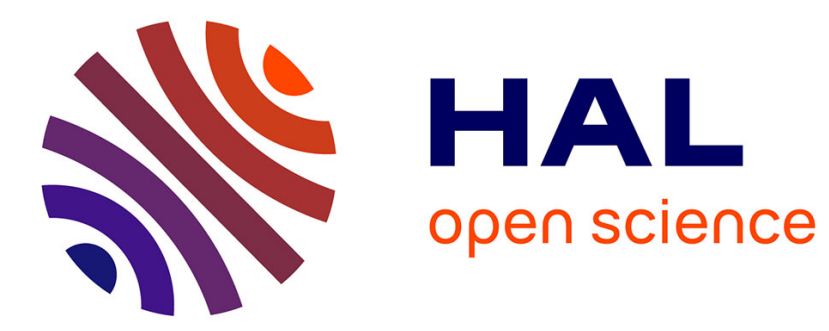

\title{
Les trois premiers chapitres d'Osée et la gestion du temps dans la manière de raconter l'alliance
}

\author{
Elena Di Pede
}

\section{To cite this version:}

Elena Di Pede. Les trois premiers chapitres d'Osée et la gestion du temps dans la manière de raconter l'alliance. H. Hausloos; D. Luciani. Temporalité et intrigue. Hommage à André Wénin, 296, Peeters, pp.117-127, 2018, Bibliotheca Ephemeridum Theologicarum Lovaniensium. hal-03564731

\section{HAL Id: hal-03564731 \\ https://hal.univ-lorraine.fr/hal-03564731}

Submitted on 10 Feb 2022

HAL is a multi-disciplinary open access archive for the deposit and dissemination of scientific research documents, whether they are published or not. The documents may come from teaching and research institutions in France or abroad, or from public or private research centers.
L'archive ouverte pluridisciplinaire HAL, est destinée au dépôt et à la diffusion de documents scientifiques de niveau recherche, publiés ou non, émanant des établissements d'enseignement et de recherche français ou étrangers, des laboratoires publics ou privés. 


\section{LES TROIS PREMIERS CHAPITRES D'OSÉE ET LA GESTION DU TEMPS DANS LA MANIÈRE DE RACONTER L'ALLIANCE}

Les livres des Prophètes Seconds sont davantage coutumiers des oracles que des récits, cela est bien connu. Il n'est donc pas toujours aisé de leur appliquer l'ensemble des outils de l'analyse narrative. C'est probablement l'une des raisons pour lesquelles au cours de sa carrière riche et dense, André Wénin s'est assez peu intéressé à ce corpus biblique. Lorsqu'il l'a fait c'est essentiellement pour deux raisons: la première parce que certains de ses doctorants l'y ont presque contraint - mais dans ce cas la condition était de s'intéresser à des récits et non d'abord à des oracles ${ }^{1}$; la seconde pour l'intérêt littéraire et théologique de certains passages. Tel est le cas pour les trois premiers chapitres du livre qui porte le nom du prophète Osée auxquels il a consacré un petit ouvrage sans prétention mais qui demeure indispensable lorsqu'on aborde la lecture de ce texte biblique ${ }^{2}$. L'étude qu'il y mène sur l'organisation structurelle et narrative de l'ensemble fait ressortir à souhait l'unité théologique et thématique fondamentale de ces chapitres qui racontent l'histoire tourmentée de l'alliance entre YHWH et son peuple de manière métaphorique, à travers l'histoire difficile du mariage entre le prophète et sa femme. Cette étude servira de point de départ à la présente contribution, dans laquelle on voudrait fournir quelques pistes pour comprendre la gestion temporelle particulière du récit du mariage - métaphorisé dans la transposition qu'il propose de la relation YнWH/Israël - et ses effets sur le lecteur. Ces chapitres en effet servent de clé de lecture au livret, mais aussi à l'ensemble des Douze, qu'Osée inaugure ${ }^{3}$.

1. André Wénin a dirigé trois thèses dans ce cadre, acceptant le défi avec plaisir et grand professionnalisme: C. LichterT, Traversée du Livre de Jonas. Structure - récit intertextualité, soutenue en 2003; E. DI PEDE, Au-delà du refus, l'espoir. Recherches sur la cohérence narrative de Jr 32-45 (TM), soutenue en 2004 et A.C. Osus, Where Is the Truth? Narrative Exegesis and the Question of True and False Prophecy in Jer 26-29 (MT), soutenue en 2006.

2. A. WénIn, Osée et Gomer, parabole de la fidélité de Dieu (Os 1-3) (CB, 9), Bruxelles, Lumen Vitae, 1998.

3. Le propos de cette contribution est entièrement synchronique et part du présupposé que la forme finale du texte reçu (TM) a du sens en elle même. Sur la question d'Os comme ouverture des Douze, voir par ex. G. BENZI, Rhetorical Analysis, Interpretation, and Location of Hosea 1-3 in Its Relation to the Twelve Prophets Scroll, dans E. Di PEDE - D. Scaiola 


\section{RAPIDE RAPPEL DE L'ORGANISATION D'ENSEMBLE}

Os 1-3 racontent l'action prophétique du mariage du prophète. Celle-ci sert de base à la métaphore nuptiale de l'alliance et la vie même d'Osée est racontée comme parole de Yнwh à son peuple (Os 1,2). L'ensemble déroule un oracle à trame narrative: une histoire qui évoque un parcours narratif dans lequel sont impliqués des personnages et l'évolution de leurs relations y est racontée. Sur le plan de la métaphore, ce qui est mis en scène c'est la fidélité de YHWH à l'alliance avec Israël et l'infidélité de ce dernier. Le tout prend la forme d'une histoire humaine d'apparence banale, qui passe par les joies et les déceptions de la vie matrimoniale et familiale ${ }^{4}$ dans laquelle on trouve les étapes suivantes: 1. le mariage; 2. la naissance des enfants; 3. un procès en divorce et 4. l'éloignement de la femme; 5. la reprise de relations positives qui impliquent un renouveau de la vie du couple (en alliance); 6. un nouveau nom donné aux enfants ${ }^{5}$. L'ensemble cependant ne se décline pas de manière suivie, allant de l'étape 1 à l'étape 6 , mais propose trois phases, trois tableaux qui évoquent chacun plusieurs de ces étapes, dont certaines évoquées plusieurs fois, avec ça et là des «ruptures manifestes». Le lecteur se trouve dès lors face à des éléments de récit présentés selon une «logique bouleversée» ${ }^{6}$, ce qui lui donne l'impression globale de lire trois fois la même situation, ou en tout cas d'être confronté trois fois à un même mouvement qui va d'une situation négative à une situation positive, bien que les accents et les points de vue mis en avant soient différents d'une fois à l'autre.

La première fois $(1,2-2,3$, tableau I), négatif et positif sont simplement juxtaposés dans un récit à la troisième personne qui met en scène les acteurs du drame: Yнwh, le prophète, sa femme et les enfants du couple. Le mariage ordonné par YHWH entre le prophète et Gomer donne lieu à la naissance de trois enfants aux noms négatifs qui indiquent métaphoriquement le rejet d'Israël (1,2-9), car ses agissements ont brisé les liens vitaux avec son dieu. Mais sans raison apparente et en complète rupture avec cette situation négative, Israël sera réhabilité, comme l'indique le

(éds), The Book of the Twelve - One Book or Many Books? Metz Conference Proceedings. 5-7 November 2015 (FAT, 2/91), Tübingen, Mohr Siebeck, 2016, 97-106.

4. Ces chapitres ont fait couler beaucoup d'encre essentiellement sur les questions concernant sa composition et son historicité. Le but n'est pas ici de trancher ces questions. On se référera pour cela aux commentaires classiques, ainsi qu'à B. RENAUD, Genèse et unité rédactionnelle de Os 2, dans RevSR 54 (1980) 1-20 et ID., Le livret d'Osée 1-3. Un travail complexe d'édition, dans RevSR 56 (1982) 159-178.

5. Cf. WÉNIN, Osée et Gomer (n. 2), p. 15.

6. Ibid., p. 11. 
retournement des noms des enfants (2,1-3), tandis que l'histoire du salut, centrée sur l'alliance, s'en trouve restaurée.

La deuxième fois $(2,4-25$, tableau II), le prophète se fait narrateur de sa propre expérience ${ }^{7}$ en prenant ses enfants à témoin des agissements de leur mère, agissements qui ont des conséquences néfastes sur les enfants eux-mêmes $(2,6)$. S'ouvre ainsi un procès en divorce dont le but est de faire revenir la femme ${ }^{8}$. Ici, le passage du négatif au positif se fait par un développement continu, décrivant ce qui rend possible le renversement et comment il s'opère. Est ainsi mise en scène l'opposition radicale entre la déclaration solennelle de répudiation de l'épouse dans le cadre d'un procès en divorce $(2,4)$ et la reprise des relations matrimoniales $(2,18)$. La transition se fait sans rupture, même apparente, car la parole du prophète et le désir de dialogue qui s'y exprime englobent à la fois la parole qui opère la rupture et celle qui amorce la réconciliation, tentant ainsi de trouver une issue positive à l'infidélité de la femme/peuple.

La troisième fois $(3,1-5$, tableau III), le lecteur est à nouveau confronté à un récit à la première personne. Le prophète raconte qu'il reçoit l'ordre d'aimer une femme de mœurs légères, comme Yнwh aime les fils d'Israël idolâtres $(3,1)$. Cette nouvelle action symbolique permet à Osée de raconter de manière concrète le passage du péché-punition au pardon-salut qui comporte une période d'abstinence des deux parties (et pas uniquement de la femme) afin de reprendre la relation sur des bases profondément renouvelées, de part et d'autre. Le passage du négatif au positif est ainsi concrètement décrit comme le passage du péché $(3,1)$ à la conversion $(3,5)$.

La manière de raconter, le point de vue ainsi que les éléments mis en valeur sont dès lors différents d'un tableau à l'autre et contribuent à façonner un portrait de la relation d'alliance dont la mise en intrigue est originale et curieuse. Elle permet d'en percevoir les étapes essentielles ainsi que les enjeux fondamentaux du désir de vie pour l'autre et de fidélité que la vie en alliance appelle.

7. Il n'est évidemment pas question ici de l'expérience historique du prophète mais de celle qui se déploie dans le monde du récit.

8. On ne discutera pas ici pour savoir s'il y a effectivement divorce ou pas au terme de ce procès. Quelle que soit l'issue du rîb, le but d'une telle procédure judiciaire est de résoudre un conflit par la parole, dans l'espoir d'une solution juste pour un retour rapide à la paix (et à l'alliance). Voir P. Bovati, Ristabilire la giustizia. Procedure, vocabolario, orientamenti (AnBib, 110), Rome, Pontificio Istituto Biblico, 1986 et ID., Vie della giustizia secondo la Bibbia. Sistema giudiziario e procedure per la riconciliazione (Biblica), Bologne, EDB, 2014. Je me permets également de renvoyer à E. Di PEDE, L'alliance chez les Prophètes (CÉ, 172), Paris, Cerf, 2015, en particulier p. 30. 


\section{LA GESTION DU TEMPS DANS LE RÉCIT «MORCELÉ» DU MARIAGE}

L'oracle-récit d'ouverture du livret d'Osée, comme tout récit, déploie un temps raconté - couvrant «la durée des événements relatés» - et un temps racontant - à savoir le «temps matériel nécessaire à l'acte de raconter» ${ }^{9}$. Le premier se cale sur le temps long de l'histoire de l'alliance entre Israël et son Dieu, suivant une disposition particulière, déterminée en partie par le second, qui occupe matériellement les trois premiers chapitres du livret, autrement dit environ un quart de l'ensemble. Regardons tout d'abord la manière dont est disposé l'ensemble pour ce qui est du temps raconté.

\section{Tableau I: l'ordre du mariage et les enfants de prostitution}

Après la situation temporelle cadre du drame (Os 1,1), l'ordre du mariage inaugure la mission prophétique d'Osée, plongeant le lecteur in medias res $(1,2)$. L'ordre de se marier avec une «femme de prostitution» ${ }^{10}$ et d'avoir des «enfants de prostitution» ${ }^{11}$ est surprenant, mais l'équivalence que YHWH établit immédiatement entre cette action ordonnée à Osée et les agissements «du pays» (v. 2b) transporte le lecteur sur le plan de la métaphore et lui indique que le récit qui va suivre est à décoder à l'aide de cette clé. L'ordre est concis mais prend le temps d'expliquer ses raisons, ce qui souligne qu'il y a urgence. Du reste, comme à confirmer celle-ci, l'enchaînement mariage, conception et enfantement du premier fils est presque expéditif: quelques mots, dont quatre verbes - le premier desquels (הלך, aller) signale l'exécution précise et immédiate de l'ordre reçu - décrivent la situation qui est ensuite entièrement orientée sur la naissance du fils. C'est alors qu'un nouvel ordre parvient au prophète, un ordre qui ralentit presque brutalement le rythme jusqu'ici très soutenu. Plus que l'enfant lui-même, ce qui semble compter c'est son nom, symbolique. À travers lui, YHWH notifie la fin de la royauté en Israël, annonce proleptique de la nécessité de renouvellement indispensable de cette institution politique essentielle dans la vie du peuple.

9. J.L. SKa - J.-P. SONNET - A. Wénin, L'analyse narrative des récits de l’Ancien Testament (CÉ, 107), Paris, Cerf, 1999, p. 128.

10. L'expression est insolite et ne permet pas de trancher: s'agit-il d'une hiérodule, d'une «simple» prostituée, ou d'une femme portée à l'infidélité? Si elle a fait couler beaucoup d'encre chez les exégètes historiens, d'un point de vue synchronique il importe avant tout de souligner le penchant de la femme à l'infidélité.

11. Cette expression peut viser des enfants que la femme aurait déjà et que le prophète va ainsi adopter (mais le récit n'en fait pas mention), ainsi que aussi ceux à naître de cette union. Le lecteur comprendra plus avant que l'expression peut également souligner le fait que les fils adoptent la conduite de leur mère et sont, comme elle, idolâtres. 
Naissent ensuite deux autres enfants dont la venue au monde s'enchaîne rapidement, le passage du temps étant comme gommé par l'urgence du message dont leurs noms seront porteurs $(1,6.8)$. On notera toutefois des petites différences dans la manière de raconter les trois naissances. On ne retiendra ici que les différences d'ordre temporel. L'enchaînement rapide des actions racontées aux vv. 3, 6 et 8 écrasent le temps de la grossesse. Mais il est un autre temps, qui reçoit un traitement différencié: c'est la période de sevrage. Absente entre l'arrivée de Yizré'el et de Lo' Ruhámâ, elle est mentionnée entre la naissance de cette dernière et celle de Lo' 'ammî $(1,8)$. Or, le nom que YHwH demande de donner au cadet est extrêmement grave: il dit le rejet et la rupture définitive de l'alliance. En mentionnant le temps du sevrage de Lo' Ruhámâ, le récit soulignerait-il que YHWH prend du temps avant de se résoudre à cette annonce ultime qui annule jusqu'à la révélation du Nom à Moïse (Ex 3,14-15)? Suggère-t-il que le temps est donné au peuple pour qu'il comprenne la gravité de la situation - gravité que soulignent le mariage d'Osée et les noms des deux premiers enfants - pour que peut-être il s'amende? Rien n'est dit de cela dans l'extrême concision du récit, mais il n'est pas impossible de le penser. Car l'immobilité supposée du peuple entraîne de fait sa fin - du moins dans un premier temps.

Sans transition, en effet, l'oracle se poursuit en renouant avec la promesse faite à Abraham (Gn 22,17), tandis que les noms symboliques négatifs des trois enfants sont retournés en leur correspondant positif. Encore une fois, c'est sur un rythme soutenu que ces trois annonces de salut s'enchaînent (2,1-3). Là aussi, il y a urgence. Urgence cette fois pour la restauration, signe que YHWH ne peut se résoudre à l'échec de la relation. Il semble impatient qu'une issue positive intervienne. Et si les annonces positives semblent plus expéditives que les négatives, pour lesquelles YHWH prend le temps d'expliquer les raisons des noms symboliques des enfants, il n'en demeure pas moins que ce sont ces dernières qui comptent pour lui. Elles sont fondamentales, bien que sans raisons apparentes, comme le souligne la simple juxtaposition entre le négatif et le positif dans ce premier tableau.

\section{Tableau II: le rîb en divorce et la réconciliation}

Si le tableau I visait essentiellement le futur du peuple par des annonces proleptiques liées au nom des enfants, de punition d'abord et de restauration ensuite, le tableau II change de genre. Dans une mise en scène quasiment théâtrale, le récit cède la place à une dramatisation par le truchement du discours en «je» du personnage du prophète qui n'est plus l'exécutant 
des ordres de YHWH, mais devient acteur dans sa propre histoire de couple qu'il raconte (vv. 4-17). Le prophète s'adresse d'abord à ses enfants, puis à sa femme (désignée à la $3^{\mathrm{e}}$ personne, excepté v. 8) dans un discours qui devient progressivement déclaration de YHWH adressée en à Israël (à partir du v. 10) et où les locuteurs se superposent et se confondent. Ainsi, le tableau II se centre sur le comportement de la femme/peuple, prenant pour point de départ le présent de la relation matrimoniale et embrasse ensuite passé et futur de l'histoire commune.

L'entame $(2,4)$ semble indiquer que du temps a passé depuis la naissance des enfants puisqu'ils sont appelés pour témoigner contre leur mère. Certes, rien n'est dit de leur âge, mais le langage oblique par lequel s'ouvre ce $r i ̂ b$ a le mérite de suggérer que les agissements de la femme n'ont pas changé depuis la première affirmation de sa prostitution $(1,2)$, malgré la naissance des enfants, les annonces de malheur dont leurs noms sont porteurs et le temps qu'ils grandissent pour pouvoir témoigner. L'attention tourne cette fois entièrement autour de la femme/peuple, dont l'histoire passée est rappelée, d'abord comme débouchant sur une punition à venir $(2,5)$, puis comme point de départ du renouveau $(2,16-17)$. Il y a donc un mouvement de va-et-vient entre passé $(2,5.7 .10 .17)$ et futur (2,5.8-9.1125). Ce dernier est déterminé par la manière dont la femme agira par rapport à ses amants, mais également par la réaction du mari qui, après la punition qui passe par l'arrêt de la production agricole et la destruction de ses moyens $(2,11.14)$ ainsi que la cessation des fêtes $(2,13)$, restaurera l'histoire commune ${ }^{12}$ qui aboutira à la restauration de l'alliance $(2,20)$ qui touchera la création tout entière. Ses effets auront des conséquences bénéfiques sur les enfants $(2,24-25)$ qui n'auront plus à pâtir des séquelles négatives que les agissements néfastes de leur mère ont sur eux $(2,6)$.

Ce tableau II se déploie sur un mode où l'urgence est en apparence moins prégnante que dans le premier. Le passage - une plaidoirie qui expose et explique longuement les raisons du procès en divorce - fait ressortir l'urgence non pas par son rythme, celui du discours où temps racontant et raconté sont pratiquement équivalents, mais par les paroles qui y sont prononcées. Celles-ci dessinent ce que l'on pourrait appeler un «temps évoqué» qui embrasse passé et avenir du peuple pour figurer ce dernier, évoquant le passé négativement en signe de punition $(2,5)$ ou positivement pour annoncer la restauration $(2,17)$ future. L'issue finale dépend en réalité des choix que la femme/peuple posera dans son présent. Ainsi, la façon dont évolue la plaidoirie manifeste que le procès ne vise pas la

12. Un double «oracle de YнWH» $(2,15.18)$, vient ponctuer la rupture entre négatif et positif et le passage de l'un à l'autre se fait en 2,16-17. 
séparation définitive car après avoir envisagé des châtiments parfois radicaux, elle se prolonge en imaginant des chemins de réconciliation qui, s'ils supposent le pardon du mari, ne font pas l'impasse sur la nécessité d'une conversion pleine et sincère de la femme ${ }^{13}$. Encore une fois, si la punition est nécessaire, le prophète - et YHWH derrière lui - ne peut se résoudre à ce qu'elle soit le mot de la fin de l'histoire commune avec sa femme (Israël). Mais pour que celle-ci puisse reprendre, il est indispensable qu'il n'y ait plus de confusion entre YHWH (le mari) et les baalim (les amants) qui doivent par conséquent être éloignés.

\section{Tableau III: le rachat de l'esclave et le nouveau mariage}

Après la plaidoirie qui occupe le tableau précédent, un nouvel ordre ramène le lecteur au début du tableau I. Le prophète raconte que YHWH l'invite encore à aimer - ou à aimer encore, selon la traduction adoptée ${ }^{14}$ une femme aimée par un autre et, de surcroît, adultère. À la différence du premier ordre, où le mariage était en quelque sorte subordonné au récit de la naissance des enfants et de leur nomination symbolique, ce qui est mis en avant ici c'est la «simple» invitation à aimer cette femme «pour témoigner de l'amour que le Seigneur porte au peuple auquel il s'est allié mais qui s'est rendu coupable d'adultère ${ }^{15}$. Comme lors du premier ordre du tableau I, le récit déploie une métaphore pour exprimer l'alliance entre YHWH et Israël $(3,1)$ : par une relation (sans doute conjugale) l'homme tente de mettre en place, par amour, les conditions nécessaires pour que la femme adultère puisse retrouver le chemin de la fidélité. Plusieurs éléments montrent que, d'un point de vue temporel, le lecteur ne se trouve pas face à une analepse qui le ramènerait aux événements du tableau I et donc au premier mariage raconté une deuxième fois. En effet, l'adverbe «encore» souligne que c'est bien d'un autre ordre qu'il s'agit. Il intervient après le procès raconté dans le tableau II et permet de réaffirmer qu'après la suppression des institutions politiques et religieuses dévoyées par les agissements du peuple $(3,4)$, les fils d'Israël chercheront à nouveau leur Dieu pour leur bien $(3,5)$. Ce tableau présente lui aussi, comme le tableau II un temps évoqué qui vise la restauration à venir.

13. Cf. L. Alonso SchöKel - J.L. Sicre Diaz, Profetas. Comentario II, Madrid, Ediciones Cristiandad, 1980, p. 863: dans ce texte le pardon précède la conversion.

14. C'est la position de l'adverbe עוֹד encore, qui est ambiguë et permet les deux traductions possibles. La ponctuation massorétique préfère la seconde option.

15. WÉNIN, Osée et Gomer (n. 2), p. 47. 


\section{RÉPÉTITIONS ET GESTION DU TEMPS POUR UNE PLUS GRANDE EFFICACITÉ NARRATIVE}

Le rapide tour d'horizon sur la manière dont l'oracle-récit déploie sa temporalité interne tend à soutenir une progression suivie de l'ensemble qui raconte la complexité de l'alliance sous les traits de la métaphore d'un amour contrarié. Temps racontant et raconté s'y combinent de manière particulière pour donner un rythme qui construit un «temps évoqué» ${ }^{16}$ bien plus long que le temps raconté et qui embrasse passé, présent (de la plaidoirie) et futur. Si les deux premiers concernent essentiellement les acteurs humains du drame, YHwH pour sa part s'en tient au futur. Ce temps évoqué se déploie dans les trois tableaux qui alternent rythme rapide et lent (I: rapide; II: lent; III: lent/rapide/lent) afin de donner mouvement au temps et à l'histoire, dans une même dynamique thématique qui va du négatif au positif, du rejet à la renaissance et à la possibilité renouvelée du vivre ensemble que l'alliance permet. Ensemble, les trois tableaux visent ce temps long et fournissent les différentes pièces du puzzle servant à comprendre la dynamique de l'alliance, dans une suite qui montre un processus toujours renouvelé de restauration par lequel est soulignée la volonté de fidélité de YHWH au peuple.

Par ces trois manières différentes de raconter un même mouvement thématique qui forme l'ensemble, le lecteur se trouve donc confronté à une forme particulière de répétition ${ }^{17}$, une sorte de «triple répétition» ${ }^{18}$ qui souligne l'importance de cette histoire et du mouvement particulier qu'elle propose, tendu entre négatif et positif, entre péché (et châtiment) et restauration (et pardon), car c'est bien de l'avenir de l'alliance qu'il s'agit. Dans les récits, la triple répétition concerne normalement un élément narratif ou scénique (mais pas thématique) qui revient trois fois dans un même récit et concerne le héros (pensons par exemple à $\mathrm{Jg}$ 20,14-48 où deux batailles dramatiques pour Israël sont suivies d'une bataille victorieuse). Dans ce type de schéma de répétition, la troisième occurrence est celle qui permet au récit d'avancer vers son issue alors que l'ensemble a pour effet d'accroître la tension dramatique ${ }^{19}$.

16. Un temps que l'on pourrait peut-être aussi définir de «prophétique».

17. Comme l'écrivent SKA - SONNET - WÉNIN, L'analyse narrative (n. 9), p. 36: «La répétition est une technique qui sollicite d'une façon toute particulière la sagacité du lecteur». C'est lui qui doit repérer la manière dont elles s'enchaînent et construisent un sens nouveau. Sur la question des répétitions on verra en particulier cet ouvrage aux pp. 35-37 et R. ALTER, L'art du récit biblique (Le livre et le rouleau, 4), Bruxelles, Lessius, 1999, en particulier pp. 123-155.

18. A. WÉNIN, Samuel, juge et prophète. Lecture narrative (CÉ, 89), Paris, Cerf, 1994, p. 22

19. Cf. ibid. 
Si l'on considère cette possible grille de lecture pour l'oracle-récit d'ouverture d'Os, certains éléments émergent. Tout d'abord la volonté affirmée du mari de continuer à aimer sa femme envers et contre tout (les trois tableaux, mais surtout les tableaux II et III), ainsi que ses enfants (tableau I et fin du II). Outre le fil rouge «amoureux», les enfants servent de «liant» à l'ensemble: ils portent un nom symbolisant l'avenir d'Israël (I), sont appelés à témoigner contre leur mère dont les agissements ont pour résultat le non-amour du père à leur égard (II, cf. 2,6), et enfin, ils doivent, comme leur mère, rester assis de nombreux jours afin de se désintoxiquer: elle de sa prostitution, eux des institutions perverties (III, cf. 3,3-4). Or, comme dans le schéma de la triple répétition, c'est justement dans ce troisième tableau que les choses se jouent subtilement.

Depuis la fin du tableau II un glissement commence à s'opérer qui se concrétise ici. Ce glissement fait peu à peu sortir de la métaphore car elle se transpose à Israël à qui s'adresse l'ensemble de l'histoire qui vient d'être racontée. Ce tableau III concrétise ce passage en revenant au peuple, à la manière dont il pervertit le pouvoir et se tourne vers les idoles. Ce sont les fils d'Israël qui, concrètement, doivent amorcer le mouvement de retour, de conversion de l'idolâtrie et à chercher leur Dieu, un Dieu qui s'est donné à connaître dans l'histoire comme un dieu patient, miséricordieux et fidèle, un dieu également empressé de restaurer un peuple qui se fourvoie. La finale du tableau III semble annoncer comme une évidence cette conversion d'Israël (cf. 3,5), sorte de fin heureuse aux déboires du couple. Mais avant cela, il va falloir prendre le temps de la conversion qui suppose d'abord une forme de destruction, déterminée par les accusations très concrètes énumérées par la suite immédiate d'Os. Pour la fin positive, le lecteur doit attendre la fin du livret (14,2-10). Ainsi, le mouvement des trois tableaux semble annoncer le mouvement d'ensemble du livret, voire de l'ensemble des Douze.

\section{QUELQUES EFFETS SUR LE LECTEUR}

Une telle gestion de la temporalité a certainement pour effet de retarder le moment de l'accusation directe que le prophète va lancer à ses destinataires. Elle retarde également le moment où le lecteur sera confronté à celle-ci, pour brosser dans un premier temps le portrait d'un Dieu aimant et fidèle qui ne se résout pas à la tendance spontanée de la femme/peuple de se détourner de lui. Si l'histoire se terminait avec le tableau III, le lecteur serait confronté à un happy end annoncé, digne du meilleur conte de fées. Or l'histoire reprend, comme da capo, au chapitre 4, avec une rupture 
presque abrupte qui quitte la métaphore pour ramener le lecteur à la réalité: le pays (cf. 1,2) est rempli de mensonge, de meurtre et d'adultère, tout y est mort (4,2-3) car ses habitants ne font ni fidélité, ni loyauté, et ils ne connaissent pas Dieu $(4,1)$. Et la liste des griefs continue et tout le monde en prend pour son grade (4,4-19). La dénonciation du péché est ensuite suivie d'une annonce de châtiment (5,8-14,1 avec plusieurs éléments de dénonciation présents) avant celle de la restauration promise (14,2-10). À la suite de l'oracle-récit qui se décline en trois tableaux, un quatrième, qui dessine le même mouvement que les précédents - péché, annonce du châtiment, restauration - occupe la suite du livret. Ce tableau IV est plus long, plus détaillé, plus concret dans la description de la perversion, mais se termine lui aussi par l'annonce positive de la restauration dont le lecteur peut espérer qu'elle sera tout aussi concrète que la perversion et le châtiment.

Dès lors, la fin du chapitre 3 apparaît comme des points de suspension qui ouvrent sur une suite dont l'issue positive est à construire et pour laquelle la connaissance de YHWH est essentielle $(4,1.6 ; 5,4 ; 6,3.6 ; 8,2$; 13,4 ; cf. 2,10.22) et son pardon indispensable (1,6, cf. 14,3). La question de la connaissance, évoquée directement par le verbe «connaître» (ידע) en 2,10 et 22, est en réalité au centre des trois tableaux initiaux car YHWH s'y donne à connaître à travers la manière dont il cherche à maintenir le lien vital entre lui et la femme/peuple. Dès lors, comment comprendre que les destinataires représentés de cette parole prophétique ne l'entendent pas de cette oreille?

Le lecteur peut en premier lieu comprendre et considérer que tout n'est pas joué mais que, comme le suggère le tableau II tout se joue dans l'aujourd'hui des destinataires de la parole - dont le lecteur fait partie. Ainsi, à la suite des trois premiers chapitres, les oracles qui décrivent le comportement scandaleux d'un peuple concret pourraient servir de repoussoir pour le lecteur. Confronté à ce scandale il serait ainsi appelé à un sursaut qui l'oblige à se positionner du côté du peuple que décrit le prophète ou du côté de celui qui veut retourner à YHWH, cherche à connaître YHWH, le miséricordieux qui relève et soigne son peuple (cf. 6,1-3).

\section{CONCLUSION}

L'oracle-récit qui ouvre le livret d'Os prend du temps et prend le temps de mettre les choses en perspective, en sollicitant la sagacité du lecteur. Par cette triple répétition qui le prépare à la lecture de la suite (Os et les Douze), il est appelé à connaître YHWH et à prendre distance par rapport 
aux agissements de ce peuple qui semble ne pas comprendre ni vouloir connaître son Dieu en vérité. Se dessine également un contraste entre l'urgence du changement et le temps pris pour l'exprimer ainsi que le temps long qui se déploie dans l'histoire. Tout cela a certainement pour but de souligner la gravité et l'urgence de la situation.

Cette urgence est soulignée en dernier ressort par la rupture entre les trois chapitres initiaux et la suite du livre. Cela montre qu'en réalité la fin du chapitre 3 n'est pas nécessairement une annonce proleptique qui, de ce fait, se réalisera comme telle. Car ce qui s'exprime c'est un espoir de voir l'histoire se terminer positivement, indiquant une voie possible - mais non unique - de conclusion qui arrivera, à n'en pas douter, mais peutêtre à plus long terme que ce que le lecteur serait en droit d'espérer. Cette manière particulière de construire cette histoire dessine le cadre global du poème et du livret: «une alliance formellement rompue est nouée à nouveau. Cette transformation a un moteur, (...) c'est la parole du mari ou de Dieu et ce qu'elle donne à penser de celui qui parle ainsi. Car il parle sans cesse. Il se répète; il accuse, menace, condamne, comme s'il ne pouvait se résigner à l'infidélité de son épouse ou d'Israël. Sans trêve, sa parole semble ainsi chercher une issue, un moyen de ramener l'infidèle. Car cette parole inlassable de qui refuse de se résigner est un signe de fidélité, et elle rend possible en dernier ressort une issue positive» ${ }^{20}$.

Université de Lorraine

Elena Di PedE

Centre de recherche Écritures (EA 3943)

UFR Sciences Humaines et Sociales - Metz

FR-57000 Metz

France

dipede5@univ-lorraine.fr

20. WéNIN, Osée et Gomer (n. 2), p. 29. 